\title{
Boundary layer solutions to functional elliptic equations
}

\section{Michel Chipot* and Francisco Julio S.A. Corrêa**}

\begin{abstract}
The goal of this paper is to study a class of nonlinear functional elliptic equations using very simple comparison principles. We first construct a nontrivial solution and then study its asymptotic behaviour when the diffusion coefficient goes to 0 .
\end{abstract}

Keywords: singular perturbations, functional equations, nonlinear elliptic problems.

Mathematical subject classification: 35A05, 35B25, 35B40, 35J60.

\section{Introduction}

Let $\Omega$ be a bounded open subset of $\mathbb{R}^{n}$. We denote by $\mathcal{A}$ a function defined on $\Omega \times L^{p}(\Omega), p \geq 1$, with values in $\mathbb{R}$ such that

$$
\begin{aligned}
& x \mapsto \mathcal{A}(x, u) \text { is measurable } \forall u \in L^{p}(\Omega), \\
& u \mapsto \mathcal{A}(x, u) \text { is continuous from } L^{p}(\Omega) \text { into } \mathbb{R}, \text { a.e. } x \in \Omega,
\end{aligned}
$$

and there exist two constants $a_{0}, a_{\infty}$ such that

$$
0<a_{0} \leq \mathcal{A}(x, u) \leq a_{\infty} \quad \text { a.e. } x \in \Omega, \quad \forall u \in L^{p}(\Omega) .
$$

If $f$ is a $C^{1}$ function vanishing at 0 and $\lambda$ a positive parameter, we are interested in finding nontrivial solutions to the problem

$$
\begin{cases}-\mathcal{A}(x, u) \Delta u=\lambda f(u) & \text { in } \Omega, \\ u=0 & \text { on } \partial \Omega .\end{cases}
$$

Received 6 March 2009.

*Partially supported by NF contract \#20-113287/1 and 20-117614/1.

** Partially supported by CNPq-Brazil-301603/2007-3. 
Here $\partial \Omega$ denotes the boundary of $\Omega$. Of course problem (1.4) is understood in a weak sense as we will see below. We will also look at the behaviour of such solution when $\lambda \rightarrow+\infty$. The nature of (1.4) is obviously non-variational in general. Problems of this type in the local framework were considered in [1], [12], [13]-[15], [17].

The paper is divided as follows. In the next section we precise our assumptions in particular on the function $f$ and give an existence result. Section 3 is devoted to examples of applications. Finally in the last section we let $\lambda$ go to $+\infty$.

\section{Existence of a nontrivial solution}

Let $\theta$ be a positive number and $f:[0, \theta] \rightarrow \mathbb{R}$ a $C^{1}$ function with the following properties

$$
\begin{aligned}
& f(0)=f(\theta)=0, \\
& f^{\prime}(0)>0, \\
& f(t)>0 \quad \forall t \in(0, \theta) .
\end{aligned}
$$

We denote by $\lambda_{1}$ the first eigenvalue for the Dirichlet problem in $\Omega$-i.e.

$$
\lambda_{1}=\operatorname{Inf}_{H_{0}^{1}(\Omega)} \frac{\int_{\Omega}|\nabla v|^{2} d x}{\int_{\Omega} v^{2} d x} .
$$

Then we have:

Theorem 2.1. Under the assumptions above i.e. (1.1)-(1.3) and (2.1)-(2.4) and if

$$
\lambda>\frac{\lambda_{1} a_{\infty}}{f^{\prime}(0)}
$$

there exists a nontrivial solution to

$$
\left\{\begin{array}{l}
-\Delta u=\frac{\lambda f(u)}{\mathcal{A}(x, u)} \quad \text { in } \Omega, \\
u \in H_{0}^{1}(\Omega),
\end{array}\right.
$$

such that $u(x) \in(0, \theta)$ for a.e. $x .((2.6)$ is of course understood in a weak sense). 
Proof. Let us denote by $\varphi_{1}$ the first normalized eigenfunction to the Dirichlet problem that is to say the function such that

$$
\left\{\begin{array}{l}
-\Delta \varphi_{1}=\lambda_{1} \varphi_{1} \quad \text { in } \Omega \\
\varphi_{1} \in H_{0}^{1}(\Omega), \varphi_{1} \geq 0, \int_{\Omega} \varphi_{1}^{2} d x=1 .
\end{array}\right.
$$

1. One can choose $t_{0}$ in such a way that $\underline{u}=t_{0} \varphi_{1}$ satisfies for every $w \in L^{p}(\Omega)$

$$
-\Delta \underline{u} \leq \frac{\lambda f(\underline{u})}{\mathcal{A}(x, w)} \quad \text { in } \Omega .
$$

Indeed, due to (2.7) we have

$$
-\Delta \underline{u}=\lambda_{1} t_{0} \varphi_{1} \quad \text { in } \Omega .
$$

Assuming $t_{0}$ small enough in such a way that $0 \leq t_{0} \varphi_{1} \leq \theta$ we have for any $w \in L^{p}(\Omega)$

$$
\lambda \frac{f\left(t_{0} \varphi_{1}\right)}{a_{\infty}} \leq \frac{\lambda f(\underline{u})}{\mathcal{A}(x, w)} .
$$

Then (2.8) will be fulfilled provided we choose $t_{0}$ such that

$$
\lambda_{1} a_{\infty} \leq \lambda \frac{f\left(t_{0} \varphi_{1}\right)}{t_{0} \varphi_{1}}
$$

which is possible since

$$
\lambda_{1} a_{\infty}<\lambda f^{\prime}(0)
$$

From now on we fix $t_{0}$ in such a way that (2.8) holds. We set

$$
K=\left\{v \in L^{2}(\Omega) \mid t_{0} \varphi_{1} \leq v \leq \theta \text { a.e. } x \in \Omega\right\} .
$$

It is clear that $K$ is a closed convex subset of $L^{2}(\Omega)$.

2. We can choose $\mu$ in such a way that

$$
g(u)=\lambda f(u)+\mu u \text { is non decreasing on }(0, \theta) .
$$

Indeed it is enough to have

$$
g^{\prime}(u)=\lambda f^{\prime}(u)+\mu \geq \lambda \operatorname{Inf}_{(0, \theta)} f^{\prime}+\mu \geq 0
$$

which is possible for $\mu>0$ large. 
We suppose in what follows that (2.10) holds. For $w \in K$ we consider then

$$
u=T w
$$

the solution to

$$
\left\{\begin{array}{l}
-\Delta u+\frac{\mu u}{\mathcal{A}(x, w)}=\frac{g(w)}{\mathcal{A}(x, w)} \quad \text { in } \Omega, \\
u \in H_{0}^{1}(\Omega) .
\end{array}\right.
$$

Note that since $w \in K, w \in L^{p}(\Omega) \forall p \geq 1$ and everything makes sense, (2.12) being understood in terms of weak formulation. By the definition of $g$ one remarks that a fixed point for $T$ is a solution to (2.6).

3. $u=T w \in K$.

Due to the monotonicity of $g$ one has

$$
\begin{aligned}
& -\Delta u+\frac{\mu u}{\mathcal{A}(x, w)}=\frac{g(w)}{\mathcal{A}(x, w)} \leq \frac{g(\theta)}{\mathcal{A}(x, w)}=-\Delta \theta+\frac{\mu \theta}{\mathcal{A}(x, w)} \quad \text { in } \Omega, \\
& -\Delta u+\frac{\mu u}{\mathcal{A}(x, w)}=\frac{g(w)}{\mathcal{A}(x, w)} \geq \frac{g(\underline{u})}{\mathcal{A}(x, w)} \geq-\Delta \underline{u}+\frac{\mu \underline{u}}{\mathcal{A}(x, w)} \quad \text { in } \Omega .
\end{aligned}
$$

(The last inequality above follows from (2.8)). Since

$$
\underline{u} \leq u \leq \theta \quad \text { on } \partial \Omega
$$

we get by the weak maximum principle

$$
\underline{u} \leq u \leq \theta \quad \text { in } \Omega
$$

i.e. $u \in K$.

4. There exists a constant $C$ independent of $w$ such that

$$
\left.|| \nabla u\right|_{2, \Omega} \leq C
$$

i.e. $u$ is bounded in $H_{0}^{1}(\Omega)$ independently of $w .|\cdot|_{2, \Omega}$ denotes the usual $L^{2}(\Omega)$ norm, $|\cdot|$ the euclidean norm.

Indeed from the weak formulation of (2.12) we derive

$$
\int_{\Omega}|\nabla u|^{2}+\frac{\mu u^{2}}{\mathcal{A}(x, w)} d x=\int_{\Omega} \frac{g(w)}{\mathcal{A}(x, w)} u d x .
$$


Denote by $M$ the bound

$$
M=\operatorname{Sup}_{(0, \theta)}|g| .
$$

From (2.14) we derive since $\mu, \mathcal{A}$ are nonnegative and by the Cauchy-Schwarz inequality

$$
\int_{\Omega}|\nabla u|^{2} d x \leq \frac{M}{a_{0}} \int_{\Omega} u d x \leq \frac{M}{a_{0}}\left(\int_{\Omega} u^{2} d x\right)^{\frac{1}{2}}|\Omega|^{\frac{1}{2}} \leq \frac{M}{a_{0}} \frac{|\Omega|^{\frac{1}{2}}}{\sqrt{\lambda_{1}}}\left(\int_{\Omega}|\nabla u|^{2}\right)^{\frac{1}{2}}
$$

(we used (2.4), $|\Omega|$ denotes the measure of $\Omega$ ). It follows that

$$
\left.|| \nabla u\right|_{2, \Omega} \leq \frac{M}{a_{0}} \frac{|\Omega|^{\frac{1}{2}}}{\sqrt{\lambda_{1}}}
$$

which is (2.13).

5. The mapping $T: K \rightarrow K$ is continuous.

$K$ is of course supposed to be endowed with the $L^{2}(\Omega)$-topology. Let $w_{n}$ be a sequence such that

$$
w_{n} \rightarrow w \text { in } L^{2}(\Omega), \quad w_{n}, w \in K .
$$

One would like to show that

$$
u_{n}=T w_{n} \rightarrow T w=u \quad \text { in } L^{2}(\Omega) .
$$

From (2.12) we easily derive

$$
-\Delta\left(u-u_{n}\right)+\frac{\mu u}{\mathcal{A}(x, w)}-\frac{\mu u_{n}}{\mathcal{A}\left(x, w_{n}\right)}=\frac{g(w)}{\mathcal{A}(x, w)}-\frac{g\left(w_{n}\right)}{\mathcal{A}\left(x, w_{n}\right)}
$$

i.e.

$$
\begin{aligned}
-\Delta\left(u-u_{n}\right)+\frac{\mu\left(u-u_{n}\right)}{\mathcal{A}(x, w)}= & \mu u_{n}\left\{\frac{1}{\mathcal{A}\left(x, w_{n}\right)}-\frac{1}{\mathcal{A}(x, w)}\right\} \\
+ & \left\{\frac{g(w)}{\mathcal{A}(x, w)}-\frac{g\left(w_{n}\right)}{\mathcal{A}\left(x, w_{n}\right)}\right\} .
\end{aligned}
$$

Up to a subsequence we deduce from (2.15) that

$$
w_{n} \rightarrow w \quad \text { a.e. } x \in \Omega \text {. }
$$


From the Lebesgue theorem since $w_{n}, w \in K$ it follows that

$$
w_{n} \rightarrow w \quad \text { in } L^{p}(\Omega) \quad \forall p \geq 1 .
$$

Then the right hand side $A_{n}$ from (2.17) is such that

$$
\left|A_{n}\right| \leq \mu \theta\left|\frac{1}{\mathcal{A}\left(x, w_{n}\right)}-\frac{1}{\mathcal{A}(x, w)}\right|+\left|\frac{g(w)}{\mathcal{A}(x, w)}-\frac{g\left(w_{n}\right)}{\mathcal{A}\left(x, w_{n}\right)}\right| .
$$

Due to (1.2) and (2.18) one has

$$
\mathcal{A}\left(x, w_{n}\right) \rightarrow \mathcal{A}(x, w) \quad \text { a.e. } x \in \Omega
$$

and by the Lebesgue theorem again it easily follows that

$$
A_{n} \rightarrow 0 \text { in } L^{2}(\Omega) .
$$

From (2.17) it follows that

$$
u_{n} \rightarrow u \quad \text { in } H_{0}^{1}(\Omega) .
$$

This shows the continuity of $T$ since the possible limit $u$ is unique.

6. End of the proof.

Due to the compactness of the embedding from $H_{0}^{1}(\Omega)$ into $L^{2}(\Omega), T$ is a compact mapping from $K$ into $K$. By the Schauder fixed point theorem it has a fixed point which is a solution to (2.6). Moreover - since $u \in K-$

$$
t_{0} \varphi_{1} \leq u(x) \leq \theta \quad \text { a.e. } x \in \Omega .
$$

This completes the proof of the theorem.

Remark 2.1. If $h$ is a function satisfying (2.1)-(2.3) the existence of a nontrivial solution to

$$
\left\{\begin{array}{l}
-\Delta u=\frac{h(u)}{\mathcal{A}(x, u)}, \\
u \in H_{0}^{1}(\Omega),
\end{array}\right.
$$

is insured provided $h^{\prime}(0)>\lambda_{1} a_{\infty}$ (just set $h=\lambda f$ ). 


\section{Examples and applications}

We consider $\theta^{\prime}<0<\theta$ and $f:\left[\theta^{\prime}, \theta\right] \rightarrow \mathbb{R}$ a $C^{1}$ function satisfying

$$
\begin{aligned}
& f\left(\theta^{\prime}\right)=f(0)=f(\theta)=0, \\
& f^{\prime}(0)>0, \\
& f(t)<0 \quad \forall t \in\left(\theta^{\prime}, 0\right), \quad f(t)>0 \quad \forall t \in(0, \theta) .
\end{aligned}
$$

Under the conditions above we have

Theorem 3.1. Assuming (1.1)-(1.3), (3.1)-(3.3) and

$$
\lambda>\frac{\lambda_{1} a_{\infty}}{f^{\prime}(0)}
$$

the problem (2.6) possesses two nontrivial solutions.

Proof. There is one nontrivial solution between 0 and $\theta$ and if $\tilde{u}$ is the nontrivial solution to

$$
-\Delta u=-\frac{\lambda f(-u)}{\mathcal{A}(x,-u)}
$$

between 0 and $-\theta^{\prime}$ then clearly $u=-\tilde{u}$ is a nontrivial solution to (2.6) between $\theta^{\prime}$ and 0 . This completes the proof of the theorem.

We turn now to examine what kind of function $\mathcal{A}$ is suitable to fulfill our assumptions. First let us consider a well known local example. Let us denote by $a(x, u)$ a Carathéodory function from $\Omega \times \mathbb{R}$ into $\mathbb{R}$ i.e. such that

$$
\begin{aligned}
x & \mapsto a(x, u) & \text { is measurable } & \forall u \in \mathbb{R}, \\
u \mapsto a(x, u) & \text { is continuous } & \text { a.e. } & x \in \Omega,
\end{aligned}
$$

satisfying for some positive constants

$$
0<a_{0} \leq a(x, u) \leq a_{\infty} \quad \text { a.e. } x \in \Omega, \quad \forall u \in \mathbb{R} .
$$

Then if for $u \in L^{2}(\Omega)$ we define

$$
\mathcal{A}(x, u)=a(x, u(x))
$$

it is clear that our assumptions (1.1)-(1.3) are fulfilled. To show (1.2) - the only perhaps non completely obvious assumption - if $u_{n} \rightarrow u$ in $L^{2}(\Omega)$ then, up to a subsequence, $u_{n}(x) \rightarrow u(x)$ a.e. $x$ which implies that $a\left(x, u_{n}(x)\right) \rightarrow$ 
$a(x, u(x))$ a.e. $x \in \Omega$. Since the possible limit is unique this convergence is not up to a subsequence and this completes the proof of our claim. Problems involving (3.7) have been considered by many authors with different techniques (see [1], [23]). Our method is also well suited to attack nonlocal problems. The first interest for nonlocal problems goes perhaps back to Kirchhoff [18] (see also [2], [20]), where he considered a nonlinear wave equation. The topic was revisited recently in particular in the framework of asymptotic behaviour of parabolic equations. For an account to these issues we refer to [3]-[8], [9]-[11], [16], [19], [21] and [24]. To address a simple case consider a Carathéodory function $a$ satisfying (3.4)-(3.6). Let $j$ be a continuous function from $L^{p}(\Omega)$ into $\mathbb{R}, p \geq 1$. Then

$$
\mathcal{A}(x, u)=a(x, j(u))
$$

fulfills the assumptions of our two preceding theorems. For $j$ one can think for instance in the case $p=1$ to

$$
j(u)=\int_{\Omega} u(x) d x
$$

if $u$ is a density of population, $j(u)$ is then just the total population. One can restrict to a subpopulation by considering

$$
j(u)=\int_{\Omega^{\prime}} u(x) d x \quad \text { where } \Omega^{\prime} \subset \Omega,
$$

of course for some higher order $p$ one can consider

$$
j(u)=|u|_{p, \Omega}^{p}=\int_{\Omega}|u|^{p} d x
$$

or variants of it. One can also mix the two dependences by setting

$$
\mathcal{A}(x, u)=a(x, u(x), j(u))
$$

where $a: \Omega \times \mathbb{R} \times \mathbb{R} \rightarrow \mathbb{R}$ is for instance a continuous function satisfying for some constants $a_{0}, a_{\infty}$

$$
0<a_{0} \leq a(x, u, v) \leq a_{\infty} \quad \forall x, u, v \in \Omega, \mathbb{R}, \mathbb{R}
$$

and $j$ a continuous mapping from $L^{2}(\Omega)$ into $\mathbb{R}$. Then, it is clear that $\mathcal{A}(x, u)$ defined by (3.10) satisfies our assumptions with $p=2$. If $\varphi$ is a one-to-one mapping from $\Omega$ into itself another nonlocal type of nonlinearity generalizing (3.7) could be

$$
\mathcal{A}(x, u)=a(x, u(\varphi(x)))
$$


This kind of problems have been addressed in [5]. The reader will of course be able to construct for himself further examples of applications.

We would like to address now the issue of asymptotic behaviour of these solutions to problem (2.6) when $\mathcal{A}$ is given by one of the case above and when $\lambda \rightarrow+\infty$.

\section{Asymptotic behaviour}

We have first the following

Lemma 4.1. Under the assumptions of Theorem 2 let $u=u_{\lambda}$ be a nontrivial solution to (2.6) as we constructed there. Then for every $p \geq 1$ one has

$$
u_{\lambda} \rightarrow \theta \quad \text { in } L_{\mathrm{loc}}^{p}(\Omega) .
$$

Proof. We have to show that for every compact subset $S \subset \Omega$ one has when $\lambda \rightarrow+\infty$

$$
u_{\lambda} \rightarrow \theta \quad \text { in } L^{p}(S)
$$

1. We show that $\frac{f\left(u_{\lambda}\right)}{\mathcal{A}\left(x, u_{\lambda}\right)} \rightarrow 0$ in $\mathcal{D}^{\prime}(\Omega)$.

As classical $\mathcal{D}^{\prime}(\Omega)$ denotes the set of distributions on $\Omega, \mathcal{D}(\Omega)$ the space of $C^{\infty}$-functions with compact support in $\Omega$. By the weak formulation of (2.6) one has

$$
\int_{\Omega} \frac{f\left(u_{\lambda}\right)}{\mathcal{A}\left(x, u_{\lambda}\right)} \varphi d x=\frac{1}{\lambda} \int_{\Omega} \nabla u_{\lambda} \nabla \varphi d x \quad \forall \varphi \in \mathcal{D}(\Omega) .
$$

By integration by parts we get

$$
\int_{\Omega} \frac{f\left(u_{\lambda}\right)}{\mathcal{A}\left(x, u_{\lambda}\right)} \varphi d x=\frac{1}{\lambda} \int_{\Omega} u_{\lambda} \Delta \varphi d x \rightarrow 0
$$

since $u_{\lambda}$ is uniformly bounded. This completes the proof of our claim.

Let $S$ be a compact subset of $\Omega$. We claim that

2. For any $\eta>0,\left|\left\{x \in S \mid u_{\lambda} \leq \theta-\eta\right\}\right| \rightarrow 0$ as $\lambda \rightarrow+\infty$.

$(|\cdot|$ denotes the measure of sets $)$.

First some remarks are necessary. It is easy to see that the choice of $t_{0}$ in the proof of Theorem 2.1 can be made independently of $\lambda$ large. Thus, since $u_{\lambda} \in K$, for every $\lambda$ (see (2.9) for the definition of $K$ ) one has

$$
\operatorname{Inf}_{S} u_{\lambda} \geq \operatorname{Inf}_{S} t_{0} \varphi_{1}=\gamma>0 .
$$


(Indeed $\varphi_{1}$ as eigenfunction of the Laplace operator is smooth in $\Omega$ and since $S$ is compact the infimum of $t_{0} \varphi_{1}$ on $S$ is achieved and positive). Note here that the constant $\gamma$ is independent of $\lambda$. Let us denote then by $\varphi$ a nonnegative function such that

$$
\varphi=1 \quad \text { on } S, \quad \varphi \in \mathcal{D}(\Omega) .
$$

We have since $f, \mathcal{A}, \varphi$ are nonnegative

$$
\int_{\Omega} \frac{f\left(u_{\lambda}\right) \varphi}{\mathcal{A}\left(x, u_{\lambda}\right)} d x \geq \int_{S} \frac{f\left(u_{\lambda}\right)}{\mathcal{A}\left(x, u_{\lambda}\right)} d x \geq \int_{\left\{u_{\lambda} \leq \theta-\eta\right\}} \frac{f\left(u_{\lambda}\right)}{a_{\infty}} d x
$$

where we have set

$$
\left\{u_{\lambda} \leq \theta-\eta\right\}=\left\{x \in S \mid u_{\lambda}(x) \leq \theta-\eta\right\} .
$$

On this set above, by (4.3), we have (see (2.3))

$$
f\left(u_{\lambda}\right) \geq \operatorname{Inf}_{(\gamma, \theta-\eta)} f=c>0 .
$$

Going back to (4.4) we deduce

$$
\int_{\Omega} \frac{f\left(u_{\lambda}\right) \varphi}{\mathcal{A}\left(x, u_{\lambda}\right)} d x \geq \frac{c}{a_{\infty}}\left|\left\{u_{\lambda} \leq \theta-\eta\right\}\right| .
$$

Claim 2 follows then from part 1 .

3. End of proof.

We have for $p \geq 1$ and with the notation (4.5) for any $\eta>0$

$\left|u_{\lambda}-\theta\right|_{p, K}^{p}=\int_{S}\left|u_{\lambda}-\theta\right|^{p} d x=\int_{\left\{u_{\lambda} \leq \theta-\eta\right\}}\left|u_{\lambda}-\theta\right|^{p} d x+\int_{\left\{u_{\lambda}>\theta-\eta\right\}}\left|u_{\lambda}-\theta\right|^{p} d x$ with an obvious notation for $\left\{u_{\lambda}>\theta-\eta\right\}$. It follows that

$$
\left|u_{\lambda}-\theta\right|_{p, K}^{p} \leq \theta^{p}\left|\left\{u_{\lambda} \leq \theta-\eta\right\}\right|+\eta^{p}|\Omega| .
$$

$\varepsilon$ given, one can first choose $\eta$ such that

$$
\eta^{p}|\Omega| \leq \frac{\varepsilon^{p}}{2}
$$

then for $\lambda$ large enough one has

$$
\theta^{p}\left|\left\{u_{\lambda} \leq \theta-\eta\right\}\right| \leq \frac{\varepsilon^{p}}{2}
$$

by step 2 . Combining these two last inequalities we have obtained for $\lambda$ large enough

$$
\left|u_{\lambda}-\theta\right|_{p, K}^{p} \leq \frac{\varepsilon^{p}}{2}+\frac{\varepsilon^{p}}{2}=\varepsilon^{p}
$$

which completes the proof of the theorem. 
Remark 4.1. With the same proof one can show that every solution $u_{\lambda}$ of (2.6) uniformly bounded from below on $S$ converges toward $\theta$ in $L^{p}(S)$ for every $p \geq 1$.

As a consequence we have

Theorem 4.1. Under the assumptions of Theorem 2.1 let $u=u_{\lambda}$ be the nontrivial solution to (2.6). Then for every $p \geq 1$ one has

$$
u_{\lambda} \rightarrow \theta \quad \text { in } L^{p}(\Omega) \text {. }
$$

Proof. Since $\Omega$ is bounded there is no loss of generality to assume $p>1$. It is clear that $u_{\lambda}$ is uniformly bounded in $L^{p}(\Omega)$. Thus, up to a subsequence, $u_{\lambda}$ is converging in $L^{p}(\Omega)$ weakly. By Lemma 4.1 it follows that

$$
u_{\lambda} \rightarrow \theta \quad \text { in } L^{p}(\Omega)
$$

By the weak lower semi-continuity of the norm and the fact that $u_{\lambda} \in(0, \theta)$ we deduce

$$
\varliminf_{\lambda \rightarrow+\infty} \int_{\Omega}\left|u_{\lambda}\right|^{p} d x \geq \int_{\Omega} \theta^{p} d x \geq \varlimsup_{\lambda \rightarrow+\infty} \int_{\Omega}\left|u_{\lambda}\right|^{p} d x .
$$

This implies that

$$
\lim _{\lambda \rightarrow+\infty} \int_{\Omega}\left|u_{\lambda}\right|^{p} d x=\int_{\Omega} \theta^{p} d x
$$

and (4.6) follows by (4.7) (see for instance [22]).

\section{References}

[1] K.J. Brown and H. Budin, On the existence of positive solutions for a class of semilinear elliptic boundary value problems. SIAM J. Math. Anal., 10(5) (1979), 875-883.

[2] G.F. Carrier, On the non-linear vibration problem of the elastic string. Quart. Appl. Math., 3 (1945), 157-165.

[3] M. Chipot, Remarks on some class of nonlocal elliptic problems. Recent advances on elliptic and parabolic issues. World Scientific (2006), 79-102.

[4] M. Chipot, Elements of nonlinear analysis. Birkhäuser Advanced Texts (2000).

[5] M. Chipot, The diffusion of a population partly driven by its preferences. A.R.M.A., 155 (2000), 237-259.

[6] M. Chipot and B. Lovat, Some remarks on non local elliptic and parabolic problems. Nonlinear Anal., 30(7) (1997), 4619-4627. 
[7] M. Chipot and B. Lovat, On the asymptotic behaviour of some nonlocal problems. Positivity (1999), 65-81.

[8] M. Chipot and J.F. Rodrigues, On a class of nonlocal nonlinear problems. Math. Model. Numer. Anal., 26(3) (1992), 447-468.

[9] F.J.S.A. Corrêa, On multiple positive solutions of positone and nonpositone problems. Abstract and Applied Analysis, 4(2) (1999), 101-108.

[10] F.J.S.A. Corrêa, On positive solutions of nonlocal and nonvariational elliptic problems. Nonlinear Anal., 59 (2004), 1147-1155.

[11] F.J.S.A. Corrêa, Silvano D.B. Menezes and J. Ferreira, On a class of problems involving a nonlocal operator. Applied Mathematics and Computation, 147 (2004), 475-489.

[12] E.N. Dancer and K. Schmitt, On positive solutions of semilinear elliptic equations. Proc. Amer. Math. Soc., 101 (1987), 445-452.

[13] D.G. de Figueiredo, On the uniqueness of positive solutions of the Dirichlet problem for $-\Delta u=\lambda \sin u$, Nonlinear P.D.E. and Appl., Collège de France Seminar, Vol. 7, Pitman (1985), 80-83.

[14] D.G. de Figueiredo, On the existence of multiple ordered solutions for nonlinear eigenvalue problems. Nonlinear Anal. TMA, 11 (1987), 481-492.

[15] D.G. de Figueiredo, M. Girardi and M. Matzeu, Semilinear elliptic equations with dependence on the gradient via mountain pass techniques. Diff. Int. Equations, 17 (2004), 119-126.

[16] J.M. Gomes and L. Sanchez, On a variational approach to some non-local boundary value problems. Applicable Analysis, 84(9) (2005), 909-925.

[17] P. Hess, On multiple positive solutions of nonlinear elliptic eigenvalue problems. Comm. P.D.E. 6, 8 (1981), 951-961.

[18] G. Kirchhoff, Mechanik. Teubner, Leipzig (1983).

[19] J. Límaco, H.R. Clark and L.A. Medeiros, Remarks on nonlinear biharmonic evolution equation of Kirchhoff type in noncylindrical domain. Int. J. Math. Math. Sci., 2003(32), June (2003), 2035-2052.

[20] J.L. Lions, Quelques Méthodes de Résolution de Problèmes aux Limites Non Linéaires. Dunod, Paris (1969).

[21] L.A. Medeiros, J. Limaco and S.B. Menezes, Vibrations of elastic strings: mathematical aspects. Part One, J. Comput. Anal. Appl., 4(2), April (2002), 91-127.

[22] F. Riesz and B.S.Z. Nagy, Leçons d'analyse fontionnelle. Gauthier-Villars (1975).

[23] M. Struwe, Variational Methods. Springer (1990).

[24] E. Sylwestrzak, Iterations for nonlocal elliptic problems. Banach Center Publications, Institute of Mathematics, Polish Academy of Sciences, Warszawa. 


\section{Michel Chipot}

Institute of Mathematics

University of Zürich

Winterthurerstrasse 190

CH-8057 Zürich

SWITZERLAND

E-mail: m.m.chipot@math.uzh.ch

\section{Francisco Julio S.A. Corrêa}

Unidade Acadêmica de Matemática e Estatística

Centro de Ciências e Tecnologia

Universidade Federal de Campina Grande

58109-970, Campina Grande, PB

BRAZIL

E-mail: fjsacorrea@gmail.com 